\title{
A Problem in the Theory of Numbere.
}

\author{
By T. H. Miller.
}

(Read 12th June. MS. received 19th January 1908.)

One of the well known properties of the number 7 is that when $\frac{1}{7}$ is reduced to a decimal, the periods of two digits are obtained to infinity by successive doubling. It is interesting to find for what other numbers this property is true.

Let $n$ be any number, and $r$ the base of notation, then

$$
\begin{aligned}
\frac{1}{n} & =\frac{2 n}{r^{2}}+\frac{4 n}{r^{4}}+\frac{8 n}{r^{6}}+\text { to infinity } \\
& =\frac{2 n}{r^{2}-2}
\end{aligned}
$$

Therefore $r^{2}=2 n^{2}+2$ where $r$ and $n$ are integers.

To find a solution of this equation, write it in the form

$$
r^{2}-2 n^{2}=2
$$

$\therefore(r+\sqrt{ } 2 . n)(r-\sqrt{ } 2 . n)=(2-\sqrt{ } 2)(2+\sqrt{ } 2)\left\{(1+\sqrt{ } 2)(1-\sqrt{ } 2\}^{2 n}\right.$ where $p$ is any integer

$$
\therefore(r+\sqrt{ } 2 . n)(r-\sqrt{ } 2 . n)
$$$$
=\left[\left\{\frac{2-\sqrt{ } 2}{2}(1+\sqrt{ } 2)^{2 p}+\frac{2+\sqrt{ } 2}{2}(1-\sqrt{ } 2)^{2 p}\right\}+\left\{\frac{2-\sqrt{ } 2}{2}(1+\sqrt{ } 2)^{2 p}-\frac{2+\sqrt{ } 2}{2}(1 \quad \sqrt{ } 2)^{2 p}\right\}\right]
$$$$
\times\left[\left\{\frac{2-\sqrt{ } 2}{2}(1+\sqrt{ } 2)^{2 p}+\frac{2+\sqrt{ } 2}{2}(1-\sqrt{ } 2)^{2 p}\right\}-\left\{\frac{2-\sqrt{ } 2}{2}(1+\sqrt{ } 2)^{2 p}-\frac{2+\sqrt{ } 2}{2}(1-\sqrt{ } 2)^{2 p}\right\}\right]
$$

Now, from the symmetry of the expression, this equation is satisfied if we make

$$
\begin{aligned}
& r=\frac{2-\sqrt{ } 2}{2}(1+\sqrt{ } 2)^{2 p}+\frac{2+\sqrt{ } 2}{2}(1-\sqrt{ } 2)^{2 p} \\
& n=\frac{2-\sqrt{ } 2}{2 \sqrt{ } 2}(1+\sqrt{ } 2)^{2 p}-\frac{2+\sqrt{ } 2}{2 \sqrt{ } 2}(1-\sqrt{ } 2)^{2 p}
\end{aligned}
$$

both expressions being integral. Now if

$$
(2-\sqrt{ } 2)(1+\sqrt{ } 2)^{2 p}=M_{p}+N_{p} \sqrt{ } 2,
$$

then $r_{p}=\mathrm{M}_{p}$ and $n_{p}=\mathrm{N}_{p}$, where $p$ may have any positive integral value. 
Expanding, and writing $\mathrm{C}_{k}^{p}$ for the number of combinations of $p$ things taken $k$ together

$$
r_{p}=(2 p-1) 2+\mathrm{C}_{3}^{2 p-1} \cdot 4+\mathrm{C}_{5}^{2 p-1} \cdot 8+\mathrm{C}_{7}^{2 p-1} \cdot 2^{4}+\ldots+(2 p-1) 2^{p-1}+2^{p} .
$$

and $n_{p}=1+\mathrm{C}_{2}^{2 p-1} \cdot 2+\mathrm{C}_{4}^{2 p-1} \cdot 2^{2}+\ldots \quad+\mathrm{C}_{2}^{2 p-1} \cdot 2^{p-2}+2^{p-1}$.

On substituting for $p$ in succession $1,2,3$, etc., we get

$$
\begin{array}{ll}
r_{1}=2, & n_{1}=1 . \\
r_{2}=10, & n_{2}=7 . \\
r_{3}=58, & n_{3}=41 . \\
r_{4}=338, & n_{1}=239 . \\
r_{5}=7970, & n_{5}=1393, \text { etc. }
\end{array}
$$

The first is an obvious illustration, as

$$
\frac{2}{2^{2}}+\frac{4}{2^{4}}+\frac{8}{2^{6}}+\text { to infinity }=\frac{1}{1} \text {. }
$$

That the third number has the same property, can be proved from the infinite geometrical progression, or may be tested by reducing $\frac{1}{41}$ to a radix fraction in scale 58 .

If we adopt the following notation for numbers : 0 to 9 to be expressed as usual by arabic figures, ten to nineteen by $t, t_{1}, t_{2}, . . t_{20}$ twenty by $\mathrm{T}$, thirty $\theta$, forty $f$, fifty $\mathrm{F}$, with subscript figures for the excess above multiples of ten ; then $f_{1}$ represents forty-one, and

$$
\frac{1}{f_{1}}=\mathrm{iT} \mathrm{T}_{4} 2 f_{8} 5 \theta_{8} t_{1} t_{8} \mathrm{~T}_{2} \theta_{4} f_{5} t_{5} \theta_{2} \theta_{1} 74 t_{4} 8 \mathrm{~T}_{8} t_{6} \mathrm{~F}_{6} \theta_{3} \mathrm{~F}_{3} 9 \mathrm{~F}_{2} t_{9} f_{6} \theta_{8} \theta_{5} \mathrm{~T}_{1} t_{2} f_{2} \mathrm{~T}_{6} \mathrm{~T}_{8} \mathrm{FF}_{3} f_{3} f_{4} \mathrm{~T}_{9} f_{1}
$$

Now $2 \times f_{1}=1 \mathrm{~T}_{4} ; 2 \times 1 \mathrm{~T}_{4}=2 f_{8}$, etc.

The number $n$ and the base $r$ are connected by various relations, such as

$$
\begin{array}{r}
n_{p}=2 r_{p-1}+3 n_{p-1}, \\
r_{p}-r_{p-1}=n_{p-1}+n_{p}, \\
r_{p}-6 r_{p-1}+r_{p-2}=0,
\end{array}
$$

from which other forms of the series for $n$ and $r$ can be obtained and some properties of $n$ proved. Thus, the number of recurring figures in $\frac{1}{n}$ is always $n-1$ and the last figure is always $n$. 